\title{
Vortex dissipation and level dynamics for the layered superconductors with impurities
}

\author{
Ayumi Fujita \\ Center for Promotion of Computational Science and Engineering (CCSE), Japan Atomic Energy \\ Research Institute, 2-2-54 Nakameguro, Meguro-ku, Tokyo 153-0061, Japan
}

(November 1, 2018)

\begin{abstract}
We study parametric level statistics of the discretized excitation spectra inside a moving vortex core in layered superconductors with impurities. The universal conductivity is evaluated numerically for the various values of rescaled vortex velocities $\kappa$ from the clean case to the dirty limit case. The random matrix theoretical prediction is verified numerically in the large $\kappa$ regime. On the contrary in the low velocity regime, we observe $\sigma_{x x} \propto \kappa^{2 / 3}$ which is consistent with the theoretical result for the super-clean case, where the energy dissipation is due to the Landau-Zener transition which takes place at the points called "avoided crossing".
\end{abstract}

02.50.Fz, 02.60.Cb, 05.70.Ln, 73.50.-h, 74.76.Db 


\section{INTRODUCTION}

The discrete energy spectra inside the vortex core in two-dimensional superconductors play an important role for transport properties in the mixed state especially in low temperatures. The energy dissipation due to the vortex motion results from the transition between these levels. The $I-V$ characteristic in superconductors has been studied by many authors. In the superclean case $\omega_{0} \tau \gg 1$, where $\omega_{0}$ is the level separation and $\tau^{-1}$ is the inverse scattering time, the dissipative conductivity has been obtained quasiclassically as $\sigma_{x x} \simeq e n_{e} /\left(B \omega_{0} \tau\right)$. [1] For the 2D superconductor in the superclean limit where there is only one impurity inside the vortex core, the energy dissipation due to the Landau-Zener transition between levels has been evaluated. The energy transfer takes place when the impurity passes through the "dangerous region", where many Landau-Zener transitions contributing to the dissipation. [2] There is also a perturbational approach for the moving impurity which is valid in the high velocity limit $v \gg v_{F}\left(\Delta / E_{F}\right)^{2}$ where $v$ is the vortex velocity and $\Delta$ is the mean level spacing. [3] The transition between time-dependent levels $E_{i}(X)$, where $X$ is the external perturbation (the position of impurities) are treated in ref. [4] in the framework of random matrix theory, and almost the same result for $\sigma_{x x}$ as the quasi-classical one is obtained.

The parametric level statistics for the energy spectra under various external perturbations provide a new perspective of transport properties in disordered quantum systems such as metallic grains or quantum dots. The Thouless formula which relates the conductance to an equilibrium quantity such as level curvatures gave the first clue to investigations of universal behaviors of level statistics. [5.6] The random matrix theory (RMT) and its classification of distributions of level spacing (Wigner-Dyson statistics), which plays a role of a fingerprint also for quantum chaotic systems, supplies a powerful tool for the study of disordered systems in the framework of level dynamics. [7,8]

There have been both theoretical and numerical studies within the framework of the RMT for the level dynamics of the disordered systems such as an ring subject to the AharonovBohm flux and non-interacting electrons in background potentials. 99 12 For the quantum chaotic systems, there are experimental studies of the microwave billiards where the Gaussian distribution of level velocities has been observed. [13 The motivation of the current study is to investigate the universal behavior of the level velocities for low energy excitation spectra inside a moving vortex core dragged through a layered superconductor over a wide region from moderately clean to dirty phase. In the presence of disorder, the Hamiltonian for the scattering quasi-particles between the excitation levels inside the vortex core becomes the Lie algebra $S p(2 N)$, and it belongs to the class $\mathrm{C}$ of the classification of the Atland-Zirnbauer. [14] The level statistics over the crossover region from the moderately clean to the dirty phase has been investigated in the framework of the RMT [15], and also verified numerically for various impurity concentration inside the core. For the energy levels far from the Fermi energy $E_{i} \gg E_{F}$ in the dirty limit case, the level statistics have been shown to be identical with that of the Gaussian unitary ensemble (GUE). [16]

In section II, we investigate the universal distribution of the level velocities for the quasiparticle spectra within the moving vortex core for different regimes which are characterized by the parameter $\omega_{0} \tau$ (or the number of impurities within the core $N_{i}$ ), with constant vortex velocity $v$. We observe that in the case of $\omega_{0} \tau \ll 1$ the Gaussian distribution of level 
velocities is observed. In the clean case, non-Gaussian distribution with an enhanced peak at $v=0$ which is the characteristic of the systems with localized wave-functions [12] is obtained.

In the diffusive time-evolution of energy spectrum within the core, the vortex velocity $v$ plays an essential role. It should be large enough that pinning is negligible, since $v$ is taken as a constant. However it is assumed to be moderately small $v \ll v_{K}$ in order to satisfy the adiabaticity of transitions between levels. The parameter $v_{K}$ is determined by the elastic mean free path and it manifests the mesoscopic nature of the level dynamics. [4

In section III, the energy dissipation for the low velocity regime is investigated and nonlinear $I-V$ characteristics are studied. We follow the same procedure as in ref. [17] in which the energy dissipation has been evaluated through iterative calculations of timedependent spectra from the Schrödinger equation for a perturbed Hamiltonian. Our result

coincides with that obtained for the superclean case in ref. [2] in the small $v$ regime. In the dirty phase $\left(\omega_{0} \tau \ll 1\right)$, our numerical result is identical to that of the random matrix theory for the Gaussian unitary ensemble with moderately large $v$.

\section{UNIVERSALITY IN LEVEL VELOCITY DISTRIBUTION}

The excitation spectrum inside the vortex core is obtained from the Bogoliubov-de Gennes equation, 18

$$
\hat{\mathcal{H}}\left(\begin{array}{l}
u \\
v
\end{array}\right)=E\left(\begin{array}{l}
u \\
v
\end{array}\right)
$$

where

$$
\hat{\mathcal{H}}=\left(\begin{array}{cc}
p^{2} / 2 m-E_{F}+V(r) & \Delta(r) \\
\Delta^{*}(r) & -p^{2} / 2 m+E_{F}-V(r)
\end{array}\right) .
$$

We consider the case for the $s$-wave gap parameter

$$
\Delta(r)=\Delta(r) e^{i \phi}
$$

where $\phi$ is the polar angle. With the absence of impurity $(V(r)=0)$, the excitation energy spectrum

$$
E_{n}^{0}=-\left(n-\frac{1}{2}\right) \hbar \omega_{0}, \quad n=0, \pm 1, \pm 2, \cdots
$$

is obtained. We set $\hbar=1$ hereafter and

$$
\begin{aligned}
\omega_{0} & =\int_{0}^{\infty}\left[\Delta(r) d r / k_{F} r\right] e^{-2 K(r)} / \int_{0}^{\infty} d r e^{-2 K(r)}, \\
K(r) & =\int_{0}^{r} d r^{\prime} \Delta\left(r^{\prime}\right) / v_{F} .
\end{aligned}
$$

If we take $\Delta(r)=\Delta \quad\left(=\Delta_{\infty}\right)$ then the level spacing becomes $\omega_{0} \sim \Delta^{2} / E_{F}$. The $n$-th eigenfunction is 


$$
\hat{\phi}_{n}=\left(\begin{array}{c}
u_{n} \\
v_{n}
\end{array}\right)=\tilde{c} e^{-K(r)}\left(\begin{array}{c}
e^{i n \phi} J_{n}\left(k_{F} r\right) \\
-e^{i(n-1) \phi} J_{n-1}\left(k_{F} r\right)
\end{array}\right),
$$

which we call Caroli-de Gennes-Matricon (CDM) basis where $J_{n}(x)$ denotes a Bessel function and $\tilde{c}$ is a normalization constant.

When the impurity scattering is present, the wave function becomes a linear combination of the CDM basis as

$$
\hat{\psi}_{n}=\sum_{m} \hat{f}_{m n} \hat{\phi}_{m}
$$

where $n$-th column of the matrix $\hat{f}$ is given by the first order perturbation as

$$
\begin{gathered}
\left(\hat{\varepsilon}-\hat{E}+\sum_{i} \hat{A}^{i}\right) \hat{f}_{n}=0, \\
\hat{\varepsilon}_{n m}=\delta_{n m} E_{n}^{0}, \\
\hat{A}_{n m}^{i}=V_{i} e^{-2 K\left(r_{i}\right)+i \phi_{i}(m-n)}\left[J_{n}\left(k_{F} r_{i}\right) J_{m}\left(k_{F} r_{i}\right)-J_{n-1}\left(k_{F} r_{i}\right) J_{m-1}\left(k_{F} r_{i}\right)\right] / \lambda_{F} \xi,
\end{gathered}
$$

with the assumption that impurity potential $V(r)$ to be a sum of short-range scattering sites $r_{i}=\left(r_{i}, \phi_{i}\right)$

$$
V(r)=\sum_{i} V_{i} \delta\left(r-r_{i}\right)
$$

The summation for $\hat{A}^{i}$ in eq. (2.9) is taken for all impurity sites and $\xi$ is the coherence length.

When the vortex is dragged within the sample due to the Lorenz force, the Hamiltonian

$$
\hat{\mathcal{H}}(X(t))=\hat{\varepsilon}+\sum_{i} \hat{A}^{i}
$$

is perturbed by changing realizations of the impurity positions within the core. The perturbation parameter $X(t)$ is the vortex coordinate and the energy levels go up and down at different times (fig.1). Then the Hamiltonian becomes time dependent with the vortex velocity $v$ fixed as a constant.

The universal Gaussian distribution of the level velocities was studied for disordered metallic rings subject to the Aharonov-Bohm flux. [11] The level dynamics become universal dependent only on the Wigner-Dyson ensemble after a rescaling of the energy $E$ and the controlling parameter $X$ as

$$
\begin{aligned}
\varepsilon(x) & =E(X) / \Delta, \\
x & =\sqrt{C(0)} X,
\end{aligned}
$$

where $\Delta$ is the mean level spacing and $C(0)$ is the normalized dispersion of level velocities,

$$
C(0)=\frac{1}{\Delta^{2}}\left\langle\left(\frac{d E_{i}}{d X}\right)^{2}\right\rangle
$$


The statistical average $\langle\cdots\rangle$ is taken over samples and $X$, i.e. over time steps as we fix the vortex velocity $v$. It was shown that the equilibrium quantity $C(0)$ is directly related to the transport (dissipative) conductivity given by Kubo formula. [9] Thus $C(0)$ is also called the universal conductance.

The density of states of quasi-particles inside the vortex core in dirty or moderately clean phase has been investigated theoretically. [19,20,15 It was also shown from the explicit diagonalization of the Hamiltonian matrix that in the dirty limit the Wigner-Dyson statistics of level distributions become identical to that of the Gaussian unitary ensemble when the levels are far from the Fermi energy. [16] Following the same procedure we evaluate the distribution of the rescaled level velocities $P\left(\dot{\varepsilon}_{i}\right)$ where $\dot{\varepsilon}_{i}$ is written as

$$
\dot{\varepsilon}_{i}=\frac{d E_{i}}{d t} \cdot \frac{1}{\Delta \sqrt{C(0)}}
$$

for various numbers of impurities $N_{i}$ within the vortex core.

The inverse scattering time is given by

$$
\tau^{-1}=2 n_{i} V_{i}^{2} m
$$

$n_{i}=N_{i} / \xi^{2}$ is the concentration of the impurities within the core of radius $\xi$, where $\xi$ is the coherence length. In the numerical calculations we set $V_{i}=1$ and $\xi k_{F}=50$. Thus from the relation $\xi \sim k_{F} / m \Delta$ we have

$$
\left(\omega_{0} \tau\right)^{-1}=N_{i} .
$$

The size of the Hamiltonian matrix $\hat{\mathcal{H}}$ is taken as $100 \times 100$. The vortex motion is restricted in $y$-direction and periodicity in this direction for the configuration of moving impurities is assumed. The discretized shift $\Delta y$ of an impurity at discretized time step $\Delta t$ is $\Delta y=\xi v \Delta t$. The rescaled level velocity $\dot{\varepsilon}_{i}$ for 100 time steps are calculated and the $P\left(\dot{\varepsilon}_{i}\right)$ for levels between $E_{10}$ to $E_{40}$ are averaged, where we denote $E_{0}$ for the Fermi Level $\left(E_{0} \equiv E_{F}\right)$. The sample averaging has been taken over 320 (480 for $N_{i}=500$ case) different initial configurations of the impurities.

In fig. 2 we show averaged distribution of the level velocities $P(\dot{\varepsilon})$ for various numbers of impurities within the core. We set $v=0.002$ and $\Delta t=1$. The inset shows the rescaled variance $C(0)$ for each energy level. The condition for the dirty phase $1 \ll\left(\lambda_{F} / \xi\right)^{2} N N_{i} \ll N$ where $N$ is the half of the size of the Hamiltonian is well satisfied in the case $N_{i}=500$ $\left(\omega_{0} \tau=0.002\right)$. In this dirty phase the Hamiltonian can be described as the random matrix and the Gaussian distribution of the level velocities are observed.

In the clean case $N_{i}=3\left(\omega_{0} \tau \simeq 0.33\right)$, the non-Gaussian behavior is found. We obtain a sharp peak at $\dot{\varepsilon}=0$ which is similar to the result of the microwave experiment for the case when the local level dynamics are concerned. [13] This non-Gaussian behavior suggests that the wave functions for the scattering quasiparticles in this regime is still localized. [12]

\section{DISSIPATIVE PROPERTY OF THE WAVE FUNCTION OF QUASI-PARTICLES INSIDE A VORTEX CORE}

We evaluate the evolution of the wave function $|\psi(t)\rangle=\left\{\left|\psi_{1}(t)\right\rangle,\left|\psi_{2}(t)\right\rangle, \ldots,\left|\psi_{2 N}(t)\right\rangle\right\}$ from the time dependent Schrödinger equation 


$$
i \frac{d}{d t}|\psi(t)\rangle=\hat{\mathcal{H}}|\psi(t)\rangle
$$

where

$$
\hat{\mathcal{H}}=\hat{\varepsilon}+\sum_{i} \hat{A}^{i}
$$

or equivalently consider time evolution of the coefficient matrix $\hat{f}$ which depends on time.

$$
i \frac{d}{d t} \hat{f}(t)=\hat{\mathcal{H}} \hat{f}(t)
$$

The initial state $\hat{f}(0)$ is assumed to be the $i$-th eigenvector of $\hat{\mathcal{H}}$ which corresponds to the $i$-th energy level $E_{i}$.

$$
\hat{f}(0)=\left\{0, \ldots, \hat{f}_{i}, \ldots, 0\right\}
$$

Then the initial wave functions are

$$
\begin{aligned}
|\psi(0)\rangle= & \left\{0, \ldots,\left|\psi_{i}\right\rangle, \ldots, 0\right\} \\
\left|\psi_{i}\right\rangle & =\sum_{m} \delta_{n i} \hat{f}_{m n} \hat{\phi}_{m}
\end{aligned}
$$

When the vortex is moving, the impurity sites $r_{i}$ within the core are modified and the Hamiltonian $\hat{\mathcal{H}}$ becomes time dependent. We investigate the time evolution of $|\psi(t)\rangle$ which is expressed in the basis $\hat{g}$ of the instantaneous Hamiltonian $\hat{\mathcal{H}}(t)$ :

$$
\begin{aligned}
\hat{\mathcal{H}}(t) \hat{g}_{n}(t) & =E_{n}(t) \hat{g}_{n}(t) \\
\left|\tilde{\psi}_{n}(t)\right\rangle & =\sum_{m} \hat{g}_{m n} \hat{\phi}_{m} \\
\hat{f}_{n} & =\sum_{m} \hat{a}_{m n}(t) \hat{g}_{m} \\
\hat{a}_{m n}(t) & =\hat{g}_{m} \cdot \hat{f}_{n}
\end{aligned}
$$

As the vortex moves at a constant velocity $v$, the time-dependent spectrum shows diffusive time-evolution. This is the characteristic behavior which suggests that consecutive Landau-Zener transitions occur incoherently. The energy transfer takes place at the points called avoided crossings, where the separation between levels is much less than the mean level spacing $\Delta$. We assume that the quantum interference term in the transition probability between the consecutive levels can be ignored. The full quantum coherent time-evolution, however, leads to localization in energy space. The condition for this assumption is that many Landau-Zener transitions contribute to the diffusion, so we may take enough statistical average. [17

Thus the occupation probability $\left|\hat{\psi}_{n}(t)\right|^{2}$ spreads diffusively as time. Following ref. [17], we define the probability distribution $\Pi_{i}(t)$ as

$$
\Pi_{i}(t)=\sum_{k}\left(\hat{g}_{k} \cdot \hat{f}_{k}\right)(k-i)^{2}
$$

$\Pi_{i}(t)$ is averaged over samples (over different configurations of initial impurity sites), and/or over many initial states $i$. The diffusive behavior of $\langle\Pi(t)>$ for small $t$ is expected to be linearly dependent on time, 


$$
\langle\Pi(t)\rangle=R t .
$$

Solving the time dependent Schrödinger equation numerically, we investigate the dependence of the diffusion constant $R$ on the rate of change of the external perturbation, i.e. on vortex velocity $v$.

The diffusive behavior is also dependent on the smoothed density of states $\rho$. In this study we assume that $\rho$ is constant as $\rho=\Delta^{-1}$ where $\Delta$ is the mean level spacing. There are two different regimes which are characterized by the small $v$ or the large $v$ respectively. The calculation of the diffusion constant $R$ for the levels far from the Fermi energy reveals the nonlinear behavior of the energy dissipation of quasi-particles inside the vortex core in the low $v$ regime.

In fig. 3 we plot the result for the clean phase $N_{i}=3$ and the dirty phase $N_{i}=200$ for various values of $v=0.001,0.002,0.005,0.01,0.02,0.05,0.1$ and 0.2 . The initial state is $E_{i}=E_{35}$ and $E_{i}=E_{10}$, respectively near the hump of $C(0)$ plot for $v=1$ case which is shown by the insets. The horizontal axis is the rescaled vortex velocity $\kappa=\sqrt{C(0)} v$. In the calculation of the numerical solution of eq.(3.3), the discrete time step is taken as $\Delta t=0.002$ and the sample averaging has been done over 32 different initial configurations of the impurities. The gradient of the dotted line which is the least square fit is 0.67 . We find that $R \propto \kappa^{\alpha}$ where $\alpha \sim 2 / 3$ for small $v$ from the fit. Since the vortex velocity is written as $v=E / B$, this result is consistent with the behavior of the dissipative part of the current density $j_{x}$ which is obtained in ref. [2] as

$$
j_{x}=\frac{a_{0} n_{\mathrm{imp}}}{\phi_{0}} \frac{E_{F}^{5 / 3}}{\Delta^{2 / 3}}\left(\frac{E}{v_{F} B}\right)^{2 / 3},
$$

where $a_{0}$ is the distance from the "dangerous region" to the vortex center and $\phi_{0}=\pi / e$ is the flux quantum. This formula is valid for $v \ll v_{F}\left(\Delta / E_{F}\right)^{2}$ and in the super-clean limit $N_{i}=1$ case. Since $C(0)$ is suppressed with the decrease of $\omega_{0} \tau$ (or increase of $N_{i}$ ), the random matrix prediction is irrelevant as well as in the large $N_{i}$ case for this low $v$ regime as expected. We also note that for larger values of $v$, we obtain larger values of $\alpha \sim 0.9$ in all dirty cases of $N_{i}=50,100$ and 200 for which we have done the calculation.

In the meanwhile for the large $v$ regime the random matrix theory predicts the diffusion constant $R$ for the perturbed Hamiltonian whose matrix elements are taken from the Gaussian unitary ensemble (this is the relevant for the current study) as

$$
R=\pi C(0) \dot{X}^{2}
$$

where $C(0)$ is given by eq.(2.16) and $\dot{X}=v$. The vortex damping coefficient $\eta$ is defined as

$$
\partial W / \partial t=\eta v^{2}
$$

where 1.h.s is the rate of the energy dissipation inside the core and $\eta$ is related to the dissipative conductivity as $\sigma_{x x}=\eta e c / \pi B$. From the quasi-classical result in the $\omega_{0} \tau \ll 1$ case, the damping coefficient $\eta$ is given by [1]

$$
\eta=\pi n_{2 D} \omega_{0} \tau
$$

where $n_{2 D}$ is the density of electrons. Thus the random matrix theory predicts [4] 


$$
C(0)=n_{2 D}\left(\omega_{0} \tau\right)
$$

In fig. 4 we plot $C(0)$ v.s. $\omega_{0} \tau$ for various $v$ values. In the large $v$ case the relation $C(0) \propto \omega_{0} \tau$ is explicitely observed.

\section{DISCUSSION}

In this study we have investigated numerically for the universal behavior of the distribution of the rescaled level velocities for the excitation spectra within the moving vortex core in layered superconductors. In the dirty phase where $\omega_{0} \tau \ll 1$ we obtain the Gaussian distribution consistent with the result of the theoretical study for the nonlinear $\sigma$ model in the various disordered quantum systems. Contrary to this, the non-Gaussian behavior which is considered as the characteristic property which manifests the localization of wave functions inside the core is observed within the clean phase $\omega_{0} \tau \sim 1$.

As to the energy dissipation within the core due to the Landau-Zener transition between levels which occurs at the points called "avoided crossings", the nonlinear dependence of the $\sigma_{x x}$ on the external perturbation (the vortex velocity) is investigated. This is explicitly related to the nonlinear $I-V$ characteristics in the mixed state in low temperature region.

The dissipation properties are classified into three different regimes characterized by two parameters, the number of impurities inside the core $N_{i}$ (or equivalently the inverse of $\omega_{0} \tau$ ), and the vortex velocity $v$. Case i)Large $v$ and large $N_{i}$ regime; in this regime the prediction of the RMT is valid and at the same time the behavior becomes consistent with those obtained from the approach within the Kubo-Greenwood formula. Case ii)Small $v$ and large $N_{i}$ regime; in this regime the nonlinear behavior of the $\sigma_{x x}$ is observed. Since the variance of the scaled level velocity $C(0)$ is suppressed as the increase of $N_{i}$, the random matrix theoretical result is irreverent in this regime no matter how $N_{i}$ becomes large. However, we consider that a crossover behavior for the dissipation properties within the core should exist in this regime, which has been found both theoretically and numerically in the case of the density of states. Thus the third regime should be the crossover regime denoted as iii)Large $N_{i}$ and moderately large $v$ regime. We consider the result of large $\alpha$ values which are obtained in fig. 4 in large $v$ case implicitly manifest this crossover behavior. The crossover properties in this regime from the super-clean result of Larkin-Ovchinikov [2] to the dirty case where RMT result should become valid is the issue of future study.

\section{ACKNOWLEDGMENTS}

The numerical calculations has been done on the HITACHI SR2201 with 48 processors at CCSE. 


\section{REFERENCES}

[1] N.B. Kopnin and V.E. Kravtsov, Zh. Eksp. Teor. Fiz. Pis'ma Red. 23, 631 (1976) [JETP Lett. 23,578 (1976)].

[2] A.I. Larkin and Yu. N. Ovchinnikov, Phys. Rev. B 57, 5457 (1997).

[3] F. Guinea and Yu. Pogorelov, Phys. Rev. Lett. 74, 462 (1995).

[4] M.V. Feigel'man and M.A. Skvortsov, Phys. Rev. Lett. 78, 2640 (1997).

[5] J. T. Edwords and D.J. Thouless, J. Phys. C 5, 807 (1972).

[6] D.J. Thouless, Phys. Rep. 13, 93 (1974).

[7] M.L. Mehta, Random Matrices (Academic Press, New York, 1991).

[8] F. Haake, Quantum Signatures of Chaos (Springer-Verlag, 2000).

[9] E. Akkermans and G. Montambaus, Phys .Rev. Lett. 68, 642 (1992).

[10] A. Szafer and B.L. Altshuler, Phys. Rev. Lett. 70, 587 (1993).

[11] B.D. Simons and B.L. Altshuler, Phys .Rev. Lett.70, 4063 (1993), B.D. Simons and B.L. Altshuler, Phys. Rev. B 48, 5422 (1993).

[12] Y.V. Fyodorov and A.D. Mirlin, Phys. Rev. B 51, 13403 (1995).

[13] M. Barth, U. Kuhl, and H.-J. Stöckmann, Phys. Rev. Lett. 82, 2026 (1999).

[14] A. Altland and M.R. Zirnbauer, Phys. Rev. B 55, 1142 (1997).

[15] E. Brézin, S. Hikami and A.I. Larkin, Phys. Rev. B 60, 3589 (1999).

[16] A. Fujita, Phys. Rev. B 62, 15190 (2000).

[17] M. Wilkinson, Phys. Rev. A 41, 4645 (1990);

M. Wilkinson and E.J. Austin, Phys. Rev. A 46, 64 (1992).

[18] C. Caroli, P.G. de Gennes, and J. Matricon, Phys. Rev. Lett. 9,307 (1964).

[19] M. A. Skvortsov, V.E. Kravtsov and M.V. Feigel'man, JETP Lett. 68, 84 (1998).

[20] A.A. Koulakov and A.I. Larkin, Phys. Rev. B 60, 14597 (1999). 


\section{FIGURES}

FIG. 1. A snap shot of the perturbed spectra inside a vortex core where the external perturbation is due to the motion of the vortex at velocity $v$. The number of impurities inside the core is $N_{i}=3$. The discretized time step is $\Delta t=0.06$ and $v=0.1$. The size of the Hamiltonian matrix is $100 \times 100$ and 10 levels above and below the Fermi level $E_{i}=0$ are shown.

FIG. 2. The averaged distribution of the level velocities $P\left(\dot{\varepsilon}_{i}\right)$ for the vortex velocity $v=0.002$ with various numbers of impurities $N_{i}$ inside the core. The solid line corresponds to the Gaussian distribution. The inset shows the dispersion of level velocities $C(0)$.

FIG. 3. Plot of dissipation constant $R$ versus the normalized vortex velocity $\kappa$ for $N_{i}=3$ and $N_{i}=200$ cases. The result for $N_{i}=200$ is shifted downwards in order to see the coincidence of the slope of the fitting lines in lower $\kappa$ regime. The time step is taken as $\Delta t=0.002$. The gradient of the dotted line is $\alpha=0.67$ (small $\kappa$ ) and the dashed line $\alpha=0.89$ (large $\kappa$ ).

FIG. 4. Graph of log-log plot of $C(0)$ vs $\omega_{0} \tau\left(=1 / N_{i}\right)$ for various values of vortex velocity $v$ for $\Delta t=1$. 


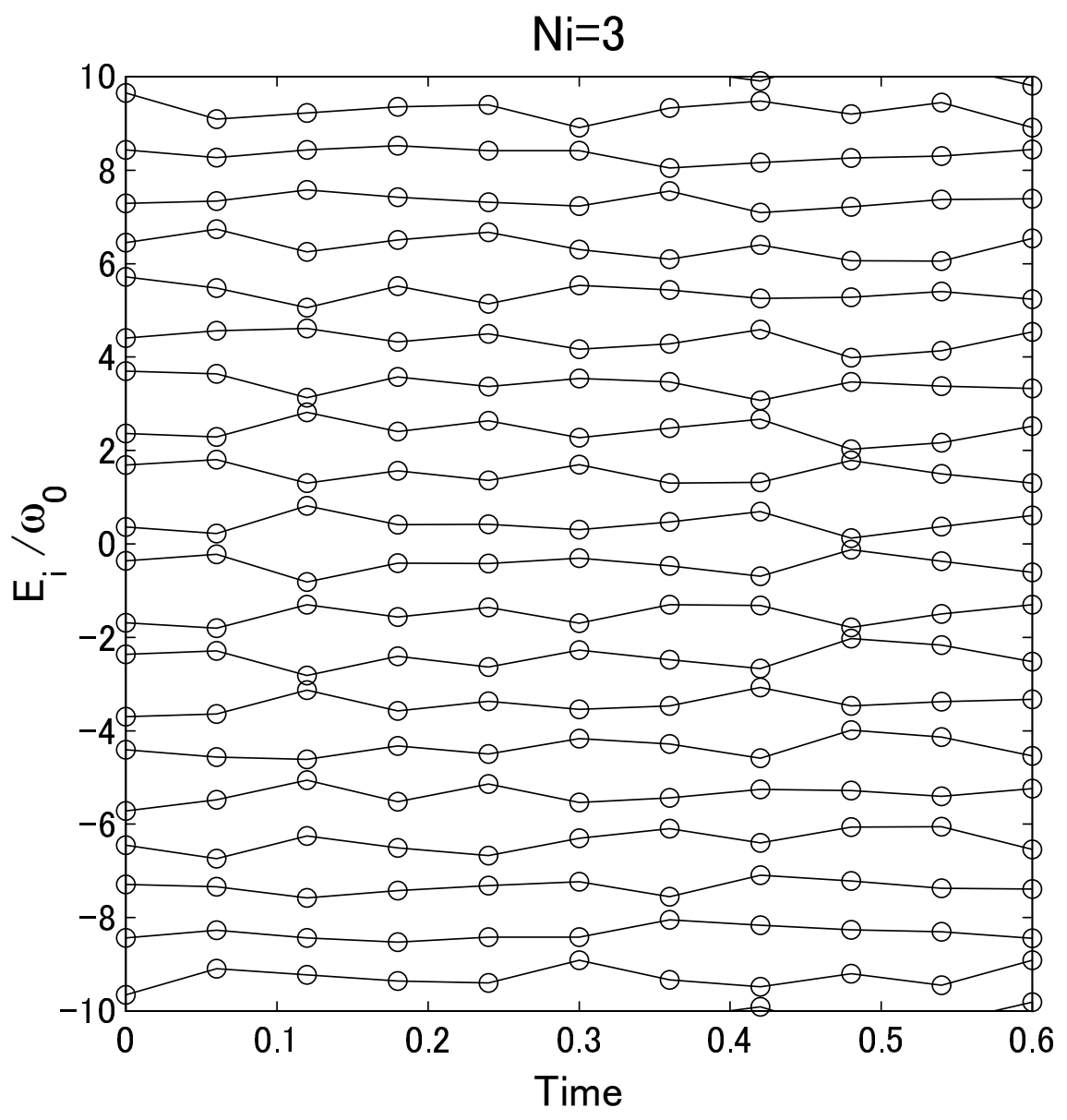




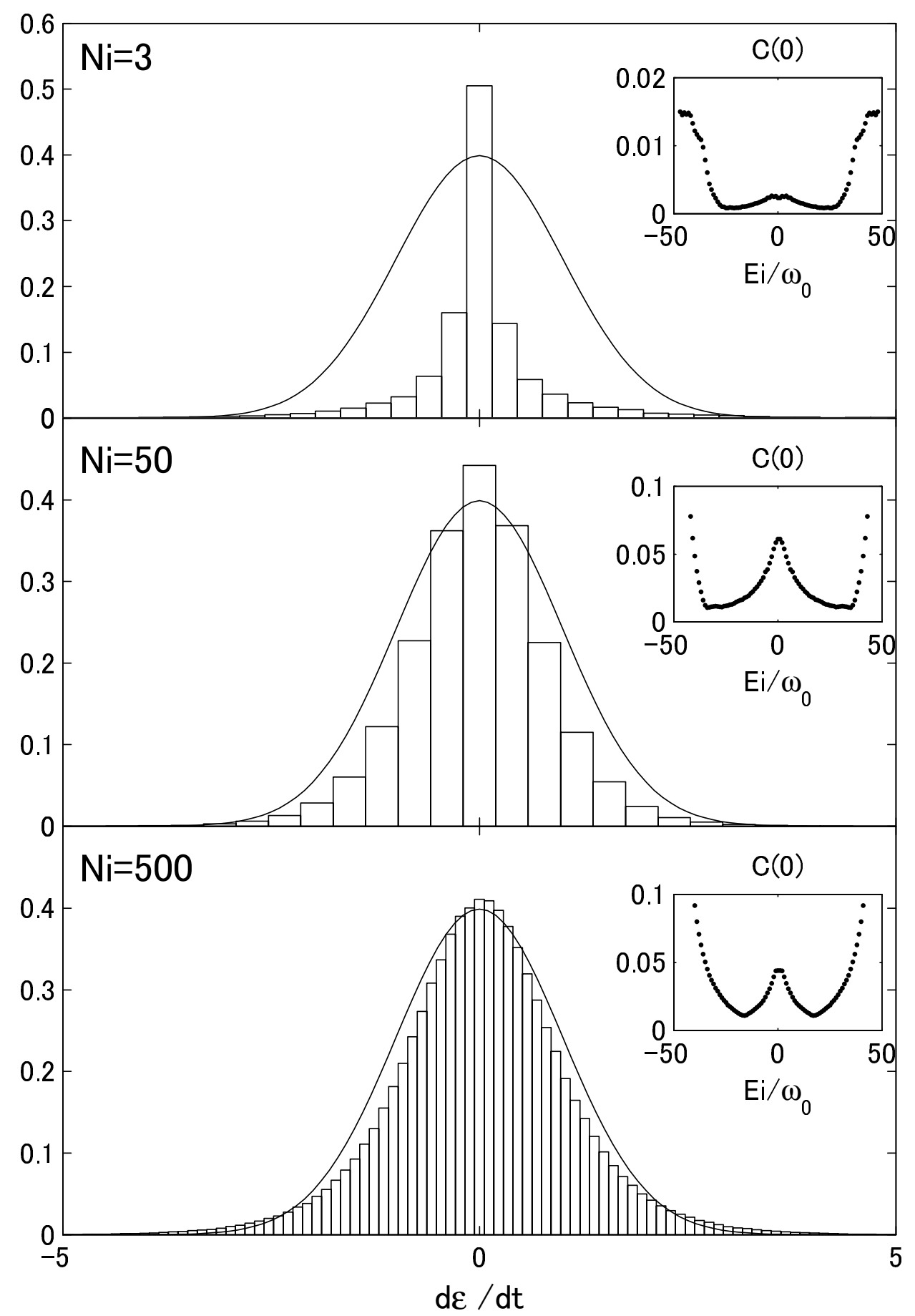




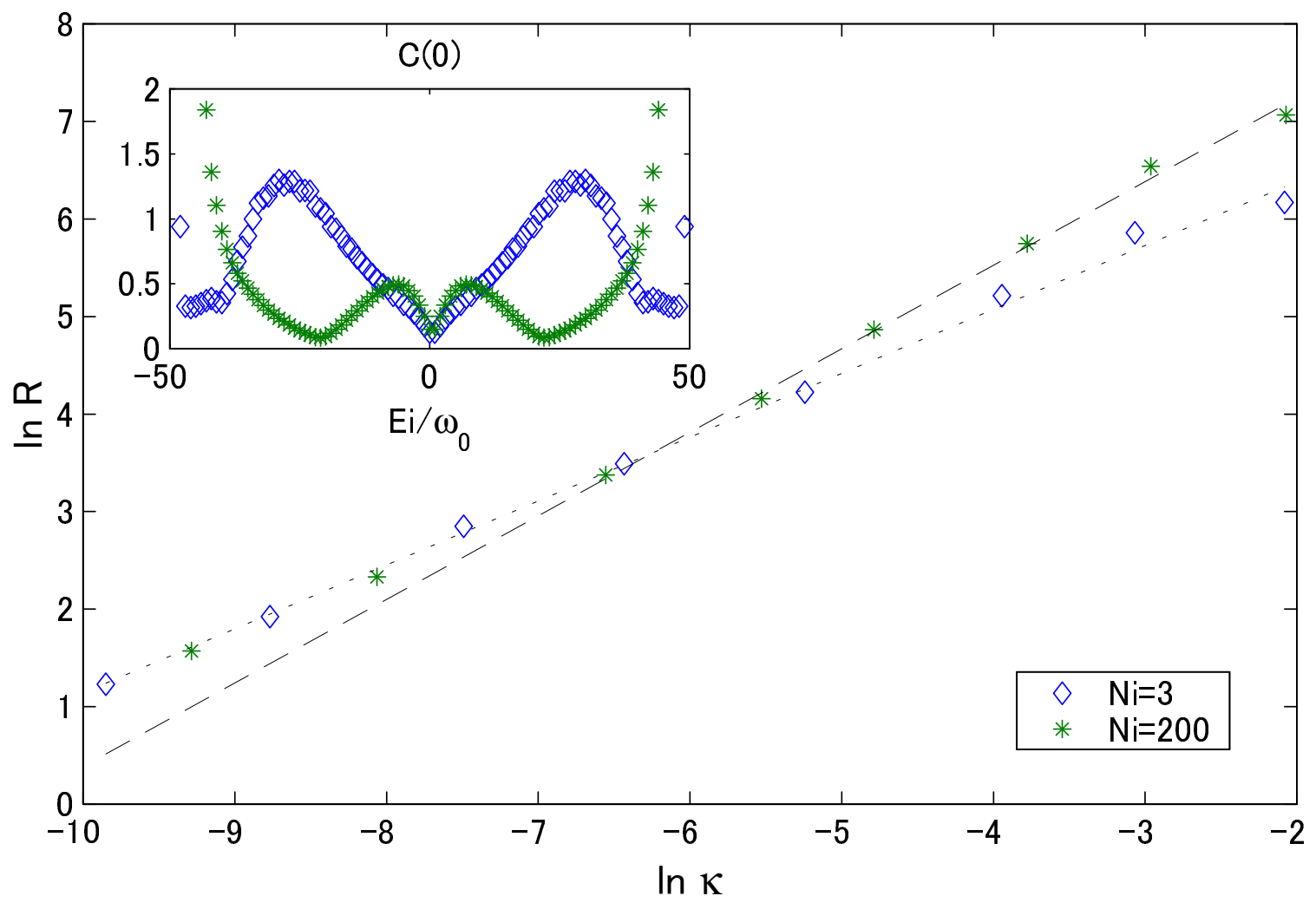




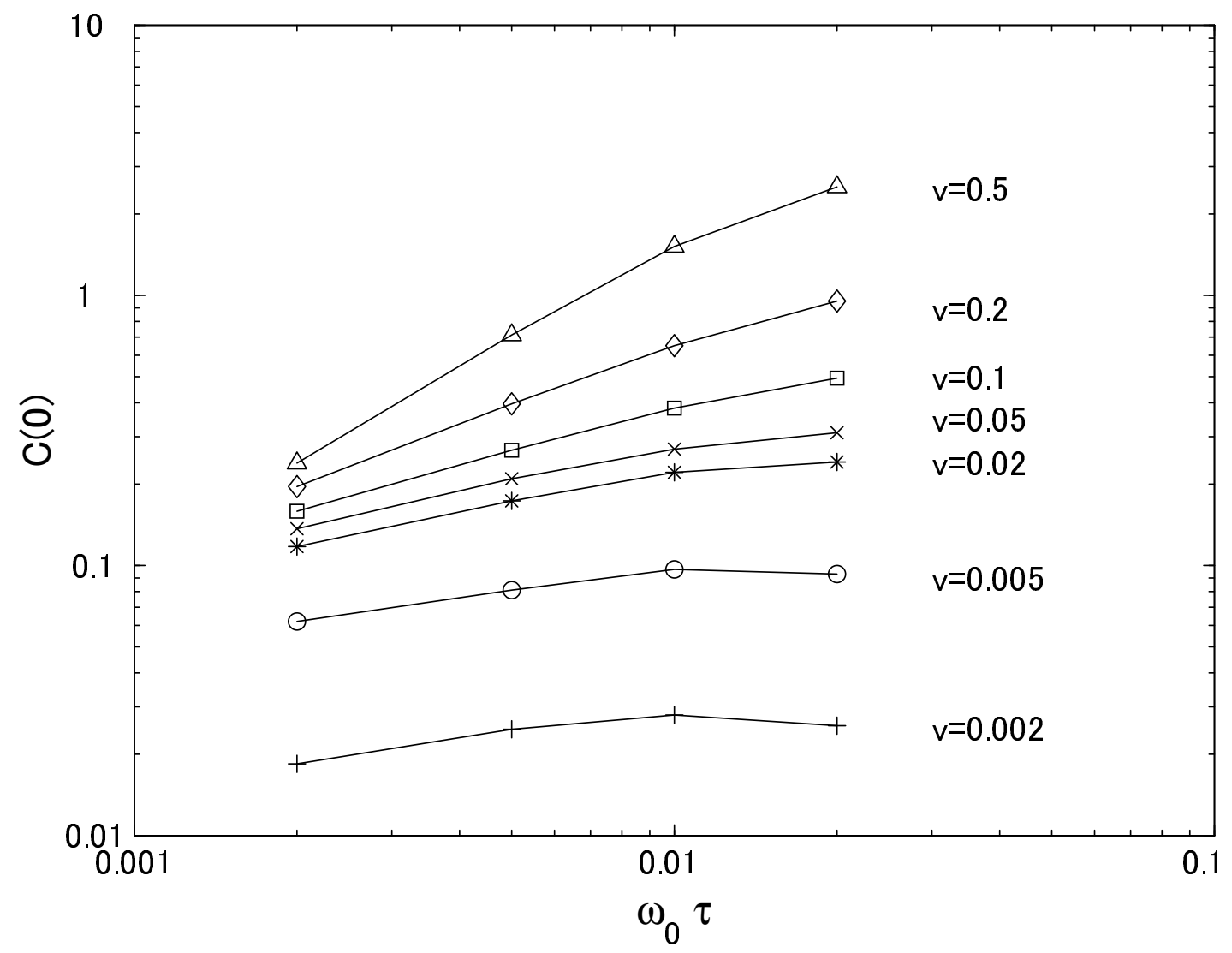

